\title{
Syneresis and vitreous hemorrhage in pseudophacia*
}

\author{
J. Reimer Wolter \\ The Departments of Ophthalmology and Pathology of the University of Michigan Hospitals, Ann Arbor, Michigan, 48109, USA
}

\begin{abstract}
The eye of a 60-year-old man with a clinically very successful lens implant became available for pathological study after a very sudden and uncomplicated death about 6 months following cataract surgery. The following changes were found: slight non-granulomatous uveitis associated with foreign-body reaction to the lens implant and its supporting iris suture, absence of the anterior vitreous face, advanced syneresis of the central vitreous, vitreous hemorrhage with diffuse layering of erythrocytes on the inner aspect of the remaining vitreous crust, vitreous traction on peripheral and central retina with tenting of foveal internal limiting membrane, and some foveal edema without cystoid changes and without foveolar detachment. Knowledge of all these changes is important, because they were compatible with good visual function and caused no clinical problems.
\end{abstract}

\section{Introduction}

Episodes of slight hemorrhaging into the vitreous are not unusual in aphacic eyes. This kind of hemorrhage can be recurrent and erythrocytes in the vitreous have the ability to survive for surprisingly long periods of time [2]. Clinically it may, thus, seem as if there is slight continuous bleeding. For example, I have clinically observed the aphacic eye of a generally healthy, elderly woman following intracapsular cataract extraction. For about 4 years during my postoperative examinations, a small layer of red blood has continuously been present in the region of a small vitreous prolapse through a round pupil. Vision and intraocular pressure remained good and no retinal holes or areas of retinal bleeding were found. However, the patient did have posterior vitreous detachment and vitreous retraction.

Small amounts of blood are also commonly seen in the vitreous of aphacic eyes containing intraocular lens implants (IOLs) [1]. The purpose of the present paper is to present a histological demonstration of the following: slight vitreous bleeding in association with aphacia, IOL, syneresis of the vitreous, slight chronic uveitis, foreign-body reaction to the implant and its supporting suture, vitreous traction on the retina, and slight edema of the central retina.

\footnotetext{
* Supported by The Research to Prevent Blindness, Inc., New York, N.Y.
}

\section{Case report}

This 60-year-old man had an intracapsular cataract extraction with implantation of a medallion type of IOL in his left eye late in 1979. There were no complications and vision was recorded as 20/20 in the eye operated upon. The patient was under treatment for hypertension, but this was well controlled. He died suddenly without warning or illness in the afternoon of 21 June 1981. An autopsy revealed sudden cardiac arrest associated with left ventricular myocardial hypertrophy and severe triple-vessel coronary atherosclerosis as the cause of death. The eyes were enucleated for the Eye Bank Program $2 \frac{1}{2} \mathrm{~h}$ after death and they were immediately placed in a refrigerator. In the morning of 22 June 1981 it was decided that the left eye with the IOL would not be used for corneal transplantation, and, thus, this eye was placed in $10 \%$ buffered formalin for fixation at about $17 \mathrm{~h}$ after death.

The eye was opened in the horinzontal plane. A clear fluid representing aqueous humor and liquefied vitreous ran out. The IOL was removed for lens implant cytology studies [6] and a cellular membrane, composed of macrophages, foreign body giant cells, so-called fibroblastlike cells, and a slightly eosinophilic acellular layer in the interspaces were found to cover the IOL. These observations have been recorded elsewhere (Figs. 1 and 2 in [7]; Fig. 7 in [8]). It is important to add that foreign-body giant cells were not only found on the IOL, but similar large giant cells with pigment granules in their protoplasm were also seen on the Prolene (polypropylene) suture used for fixation of the implant to the iris (Fig. 1).

When the IOL was removed, it was observed that the anterior vitreous face was absent. The posterior aspect of the IOL, together with the iris supporting the implant, had been in contact with the liquefied central vitreous. Histological study after paraffin embedding, sectioning, and $\mathrm{H}$ and E staining supported this gross impression and showed peripheral parts of the anterior vitreous face to extend from the pars plana of the ciliary body forward and to insert in an area slightly posterior to the iris root. Preserved zonular fiber stumps were incorporated in this membrane and this exerted forward pull on the peripheral retina, resulting in a retinal traction fold running parallel to the ora serrata (Fig. 2). Furthermore, "a crust of more resistant vitreous layers" [3] was preserved all over the surface of the retina. This was firmly attached to the retina and, thus, there was no posterior vitreous detachment. 


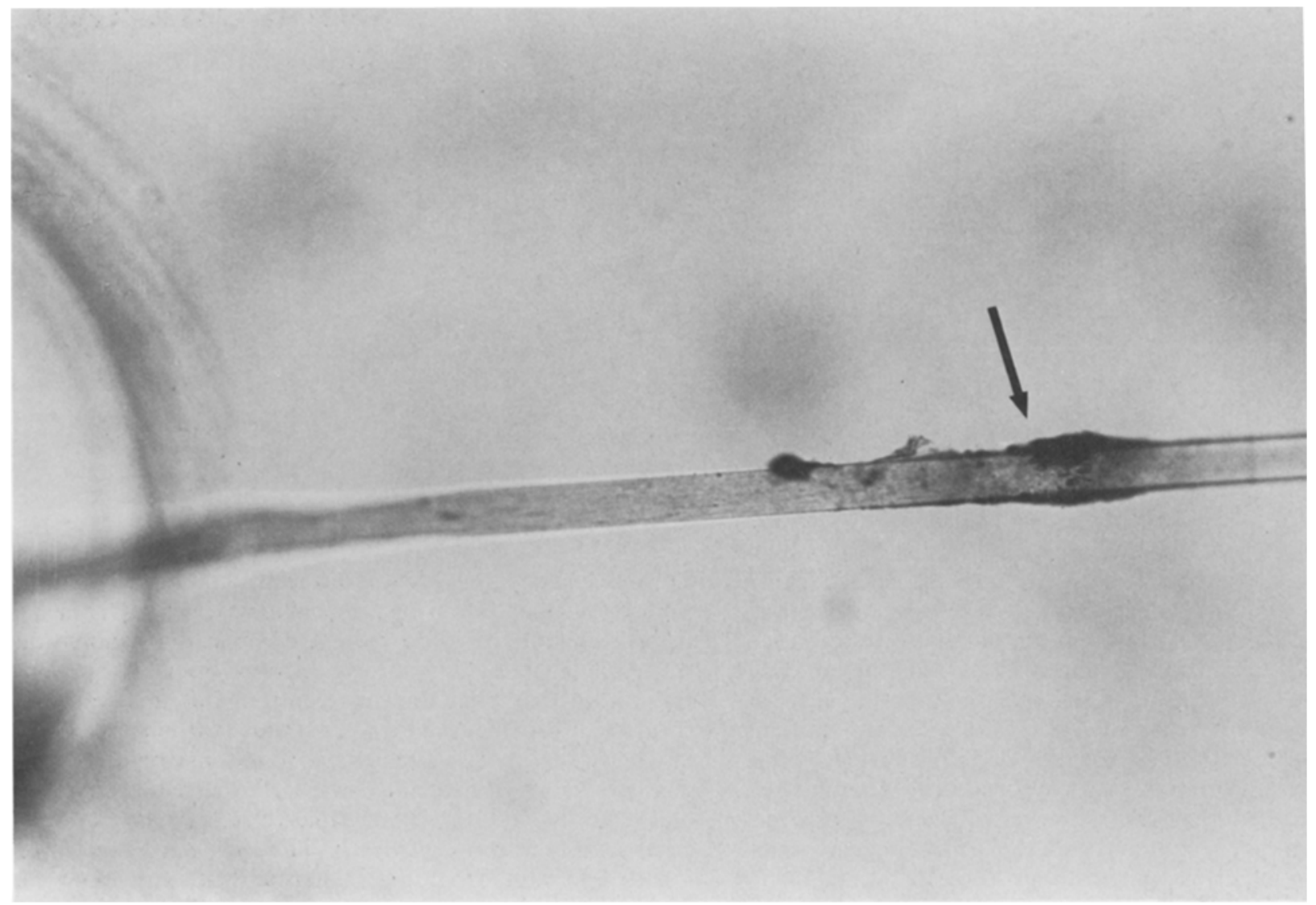

Fig. 1. Large multinucleated foreign-body giant cell with pigment granules in its protoplasm (arrow) ensheathing a portion of the Prolene iris suture: one of two suture holes in the implant on the left. Implant cytology technique, $\mathrm{H}$ and E stain, photomicrograph $\times 70$

Erythrocytes were accumulated in a thin and rather regular layer all over the inner surface of this " peripheral vitreous crust" that had remained attached to the retina (Figs. 2-4 and 6). This thin layer of very well preserved red blood cells was virtually continuous and there were, surprisingly, no associated macrophages seen. An iron stain for hemosiderin was negative - not only in the preserved layer of formed vitreous, but also in the trabeculum, ciliary body, retina, and optic nerve. The structure of the preserved vitreous crust lining the retina appeared to be denser than normal vitreous, and it histologically exhibited fibrillar and membranous formations.

Histological study of the foveal retina revealed signs of traction exerted on the central retina by the remaining outer vitreous crust. This had caused localized parafoveolar separation and tenting of the inner limiting membrane (Figs. 3-6). Traces of serous exudate seen in the space between the detached inner limiting membrane and retina (Figs. 5 and 6) indicated that this detachment was certainly not an artifact. Additional separation between the posterior vitreous face and the tented inner limiting membrane was seen in a few small areas (Figs. 5 and 6). Much swelling of the inner layers of the foveal retina with formation of a deep vertical fold running through the foveola (Figs. 3, 4, and 6) could partly be a postmortem change, but this was associated with exudate in the parafoveolar retroretinal space, indicating very slight parafoveolar exudative detachment (Figs. 3, 4, and 6). This exudate cannot be explained as an artifact. Henle's fiber layer was swollen and exhibited separation of its fibers by extracellular fluid. Henle's fibers were "standing up." The inner plexiform layer of the parafoveolar zone was also swollen due to accumulation of extra-cellular fluid (Figs. 3, 4, and 6). The central cones were in place, however, and in contact with a normal pigment epithelium (Fig. 4). It is important to emphasize that the layer of the preserved vitreous crust covering the foveal region was thin and that the erythrocytes spread out on the inner surface of this crust were very close to the foveolar retina (Fig. 6). The remainder of the histological examination of this eye revealed accumulations of mononuclear inflammatory cells in the region of the ciliary body and iris root with one larger focus of mononuclear cells in one iris process. Additional diffuse mononuclear infiltration was seen in the iris and choroid.

\section{Discussion}

Descriptive recordings of newly observed histophathological changes in single human eyes, at all stages following new surgical procedures, are essential as landmarks for a more continuous survey of these changes in the future. This reporting can serve as a preliminary guide for the surgeon in questions about the limits of ocular tolerance and adaptability to new procedures and implants. Vitreous hemorrhage has been used in the past as an example of "the invasion of the vitreous by endogenous elements, that are foreign to the vitreous itself" [3] and this can result in degeneration. Syneresis of the vitreous has been described as "one or more areas of central degeneration and liquefaction of the vitreous body, that may occur with or without 


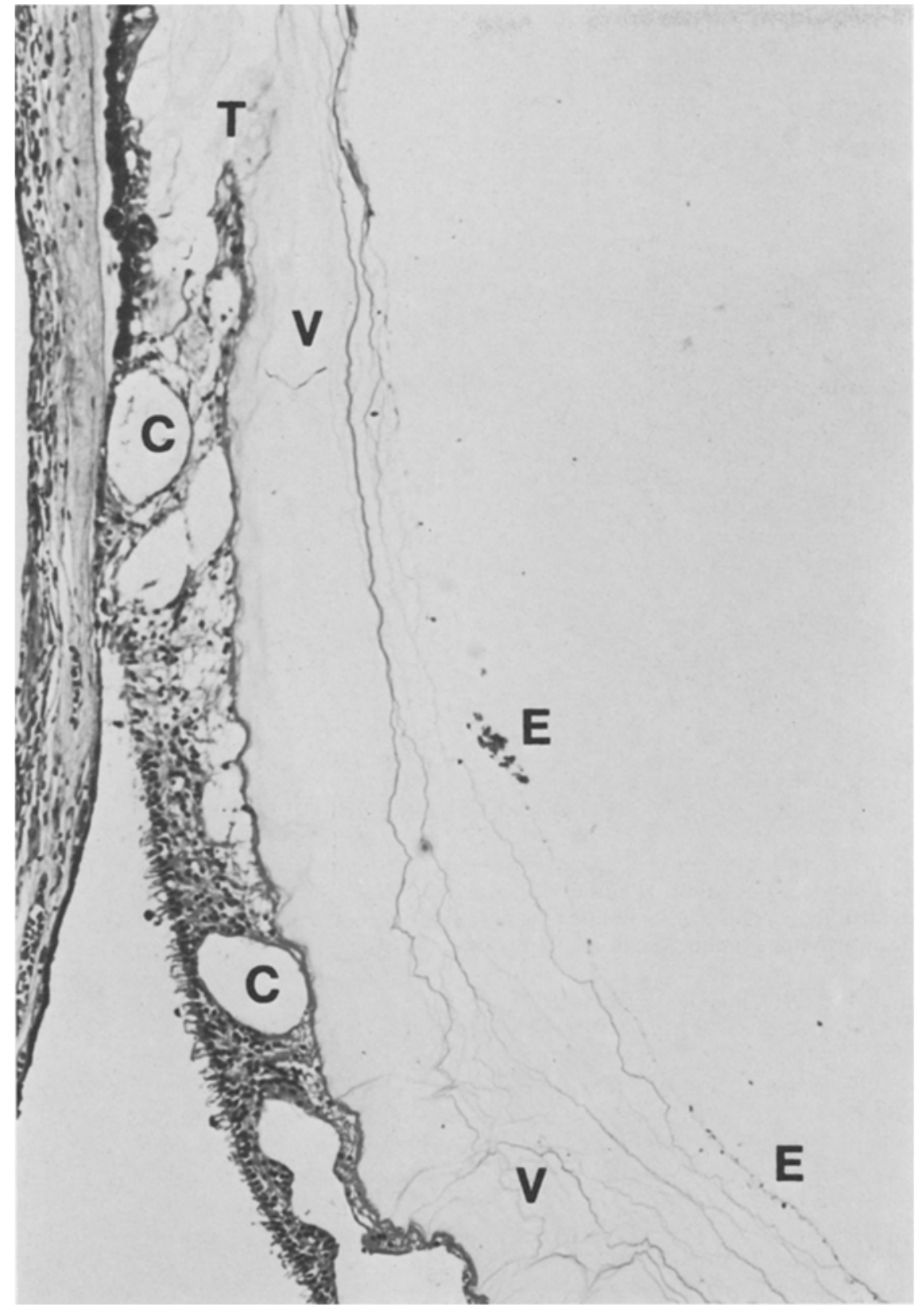

Fig. 2. Ora serrata with traction fold $(T)$. Much cystoid degeneration ( $C$ ) of peripheral retina. The formed peripheral vitreous is represented by a thin crust with strands and membranes $(V)$. Erythrocytes $(E)$ are lining the inner surface of this crust. Paraffin section, $H$ and $E$ stain, photomicrograph $\times 120$ posterior vitreous detachment" [13]. In the eye under discussion, advanced syneresis without posterior vitreous detachment was present about 6 months after cataract surgery and lens implantation. This was associated with loss of the anterior vitreous face. The remaining crust of slightly condensed vitreous, thus, was firmly attached to the retina and pars plana. There were indications that the remaining layers of vitreous were exerting forward traction on the retina in the regions of the ora serrata and the fovea. The liquefied vitreous core was in open communication with the space around the medallion lens implant occupying the pupillary zone. Distinct signs of foreign-body reaction and complete encapsulation were found on the plastic implant and its supporting suture. The uvea, furthermore, exhibited evidence of definite chronic non-granulomatous uveitis.

The thin and continuous layer of blood all over the inner surface of the remaining crust of formed vitreous in the present eye with a clinically successful IOL is a remark- able observation. The origin of this blood is not clear from our study, but it is obvious that this could very well have come from a contact area between the iris and IOL. In the absence of an anterior hyaloid membrane, the blood could have run backward into the vitreous space and accumulated on the inner surface of the crust of formed vitreous. The example of continuing vitreous hemorrhage after intracapsular cataract extraction with an intact vitreous face, as given in the introduction, shows that this type of bleeding can certainly also originate in the posterior segment and does not have to come from the anterior chamber.

Erythrocytes on the surface of lens implants are quickly phagocytized by the fibroblastlike cells (sessile macrophages) in the membranes which typically cover their surfaces $[11,12]$. Erythrocytes in the vitreous, in contrast, typically have a long lifetime [2] and only their remnants are phagocytized, when they degenerate $[4,9]$. It is important to emphasize that the presence of erythrocytes in the vitre- 


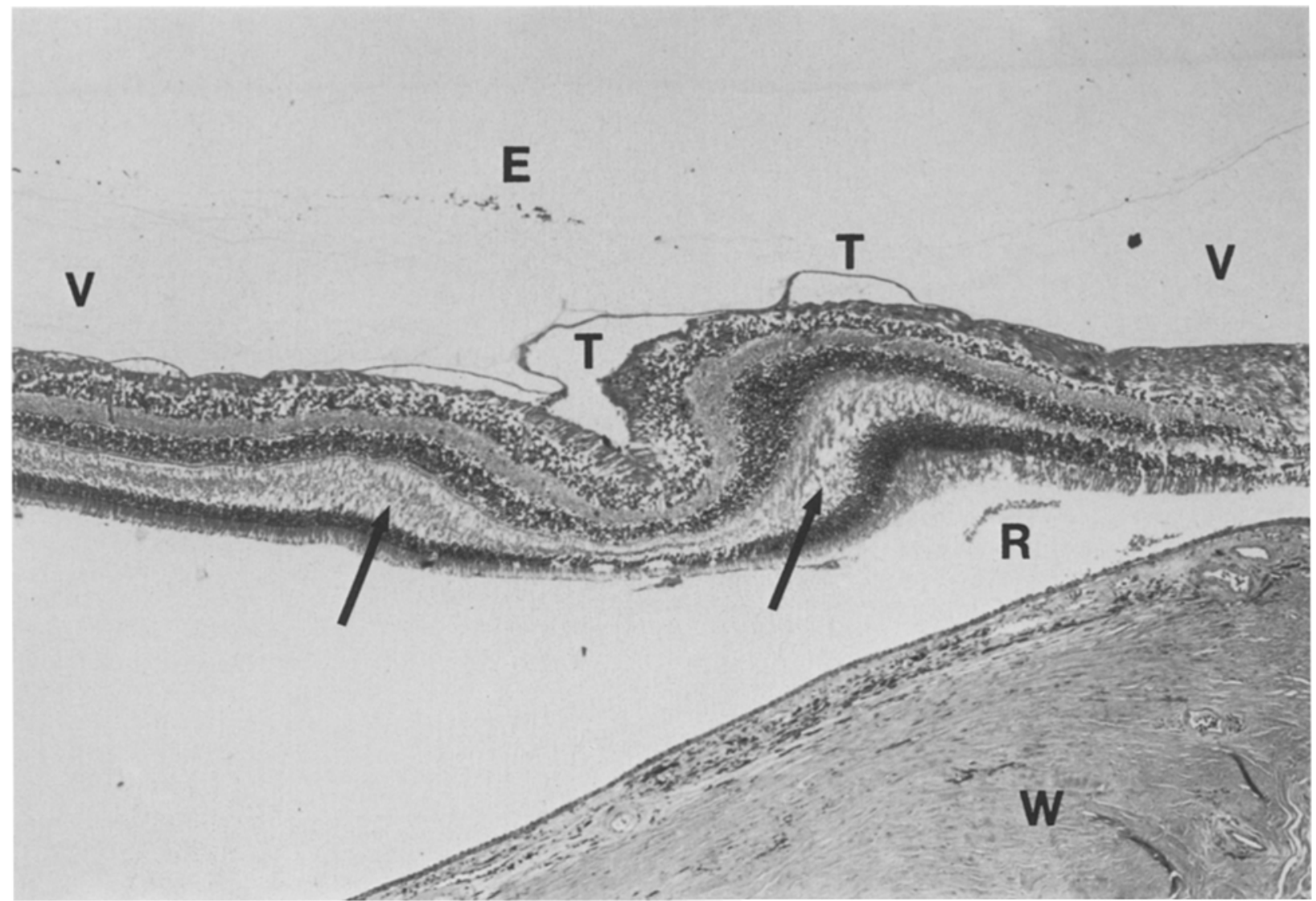

Fig. 3. Parafoveal section in a horizontal plane showing erythrocytes $(E)$ lining the inner surface of the remaining crust of formed vitreous $(V)$. The foveal retina exhibits extracellular fluid accumulation that is most extensive in Henle's fiber layer (arrows) and some parafoveolar retroretinal exudate $(\mathrm{R})$. Separation and tenting $(T)$ of the inner-limiting membrane are seen in association with the posterior vitreous face. The ocular wall $(W)$ is seen below. Paraffin section, $H$ and $\mathrm{E}$ stain, photomicrograph $\times 70$

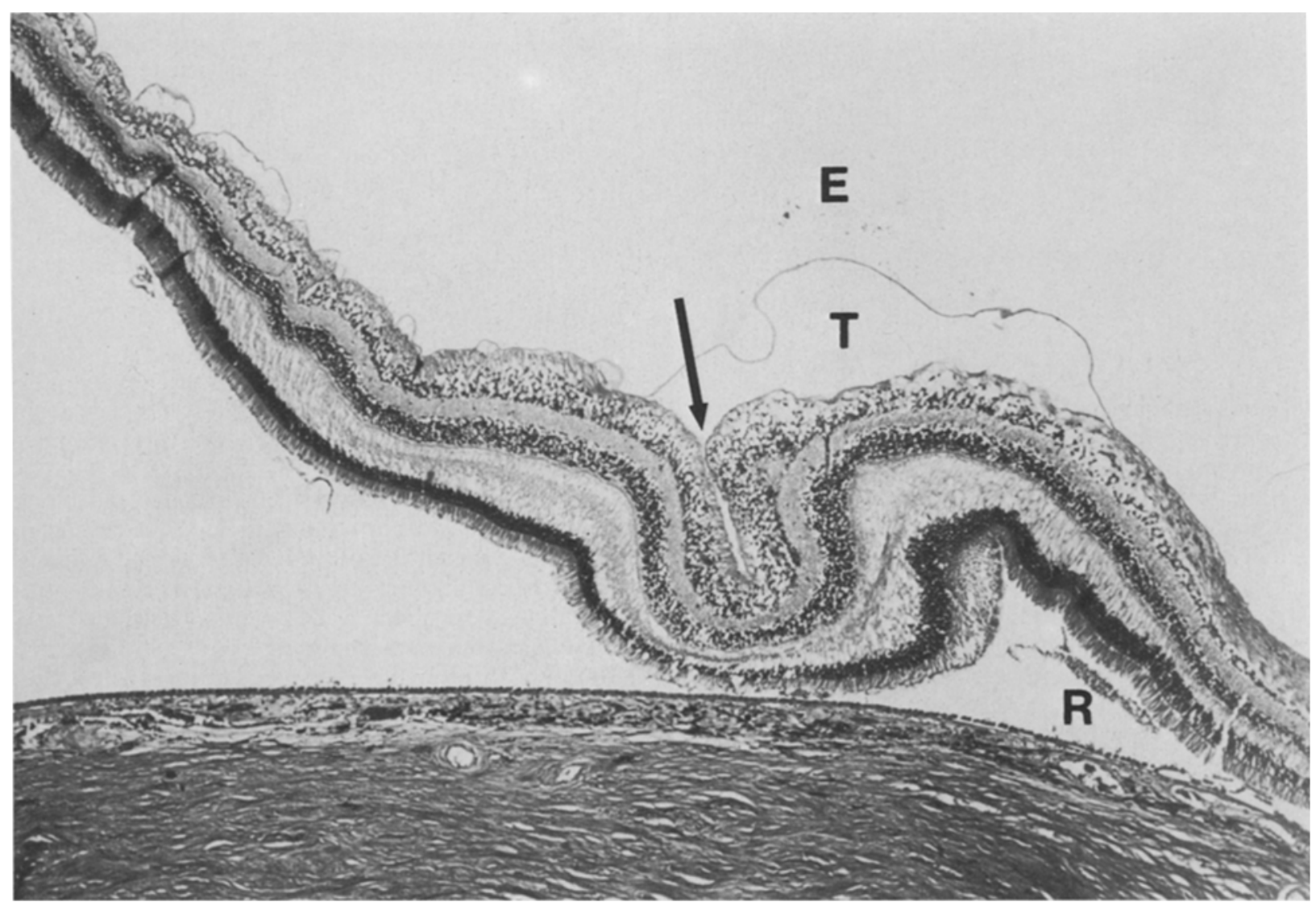

Fig. 4. Central retina in slightly parafoveolar horizontal section with erythrocytes $(E)$ in front of the fovea, a deep vertical retinal fold (arrow), tenting of the inner limiting membrane $(T)$, and slight parafoveolar retroretinal exudate $(R)$. Paraffin section, $\mathrm{H}$ and E stain, photomicrograph $\times 70$ 


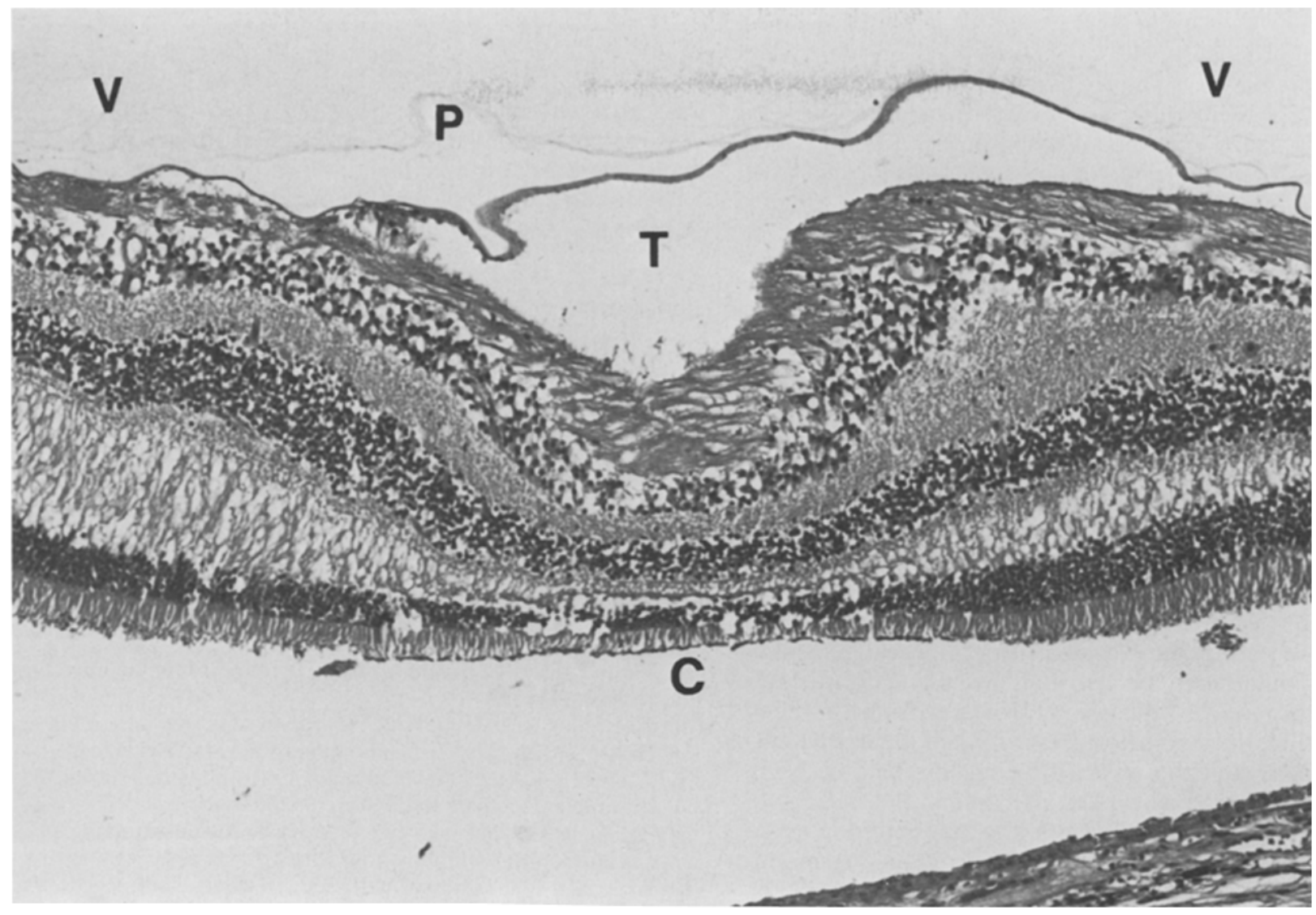

Fig. 5. Foveal retina in horizontal section with small area of separation of posterior vitreous face $(P)$ from inner limiting membrane, additional tenting of the inner-limiting membrane $(T)$, swelling of all retinal layers, and very well preserved cones $(C)$, which are artificially separated from the pigment epithelium. Vitreous $(V)$ seen above. Paraffin section, $\mathrm{H}$ and $\mathrm{E}$ stain, photomicrograph $\times 250$

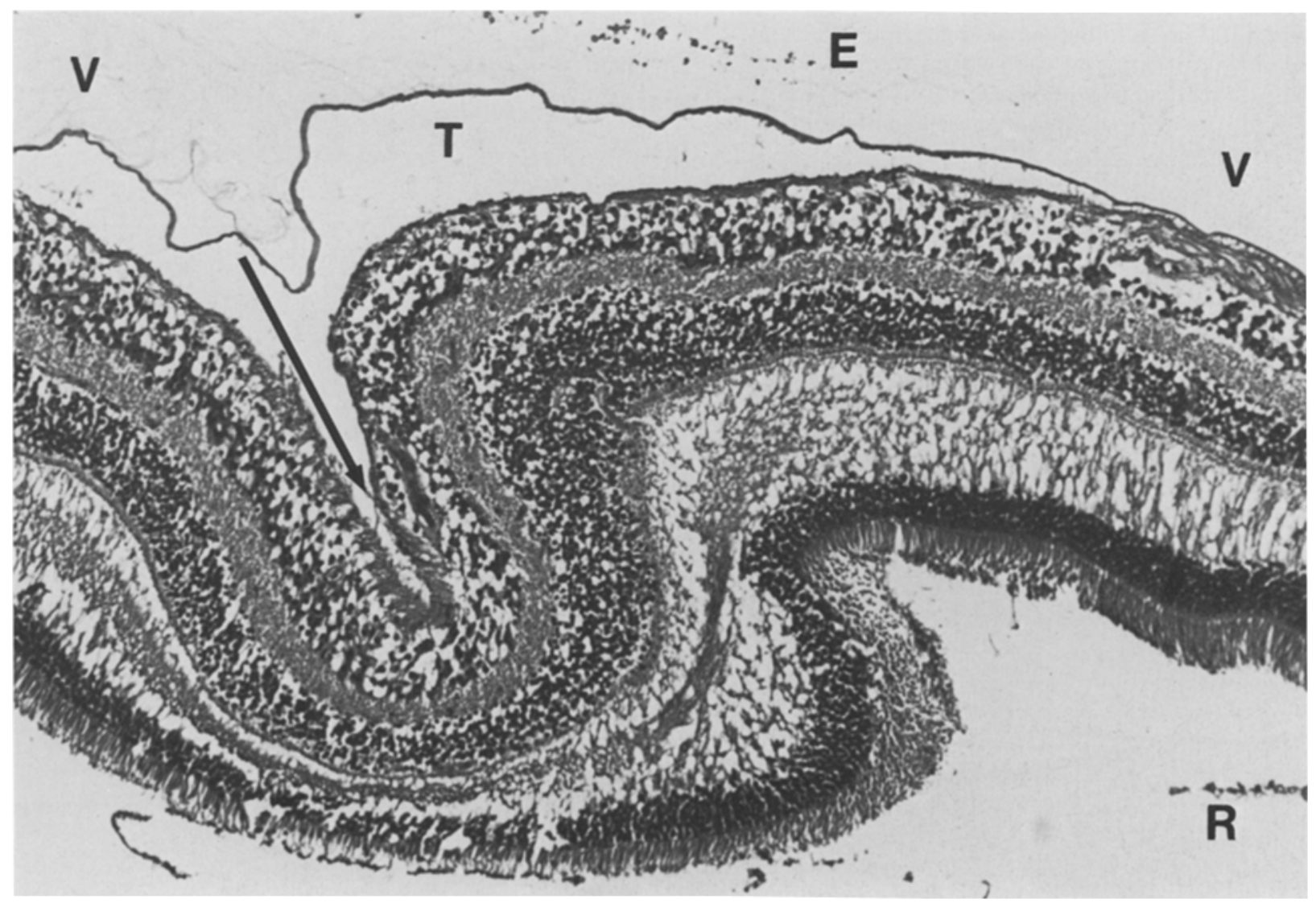

Fig. 6. Another horizontal section through the fovea showing erythrocytes $(E)$ on the vitreous crust $(V)$ very close to the tented innerlimiting membrane $(T)$. A deep vertical fold (arrow) runs through the central retina, and some exudate $(R)$ is found in the parafoveolar retroretinal space. Paraffin section, $\mathrm{H}$ and $\mathrm{E}$ stain, photomicrograph $\times 250$ 
ous of the present case was not associated with hemosiderosis - nor were there clinically obvious problems. This fits the view that small amounts of blood in the vitreous under different postoperative conditions - including aphacia with and without IOL - are not a serious problem for all practical purposes. However, it has been found that "the presence of blood exerted severe destructive effects on the gel structure of the vitreous" and that "this included liquefaction and formation of prominent bands" [1]. In the present case the remaining vitreous crust was clearly condensed and exerted traction on the foveal retina. The fact that blood was present in the vitreous will, thus, have to be on our list of possible causes - along with chronic inflammation, foreign-body reaction, vitreous traction, and hypotony - when we look for causes of vitreous degeneration and fluid leakage in the foveal retina.

The eye in the case presented had $20 / 20$ vision, the foveolar retina was in place, and the central cones were in good shape: this eye did not have cystoid macular edema (CME). The pathological changes in the fovea were only slight and could partly be artificial. The changes are compatible with good central vision. However, some swelling and exudation, the separation and tenting of the inner-limiting membrane, and the vertical folding for the time being have to be classified as typical precursors of CME [5, 9, 10]. The type of separation seen in the inner limiting membrane is well demonstrated in the Textbook on Ocular $\mathrm{Pa}$ thology by Yanoff and Fine [13] in Fig. 12-6 on page 590. These authors call the separation process of the inner-limiting membrane "tenting up of the retina at the point of attachment" of vitreous traction. In CME cases this tenting phenomenon is commonly associated with the accumulation of a loose exudate under the separated membranes. It is suspected that the cellophane-like membrane that may be clinically visible in front of the swollen fovea in some cases of CME is identical with the posterior hyaloid surface and retinal limitans interna separated as a unit from the remainder of the retina.
The selected case report on this eye with clinically successful pseudophacia presents certain new and important histopathological details. Step by step, these details will have to be understood in their full complexity, and true progress will only be possible on the basis of this understanding.

\section{References}

1. Forrester JV, Lee WL, Williamson J (1978) The pathology of vitreous hemorrhage: I. AMA Arch Ophthalmol 96:703-710

2. Samuels B (1930) Opacities of the vitreous. AMA Arch Opthalmol 4:838-857

3. Schepens ChL (1979) Clinical aspects of pathologic changes in the vitreous body. In: Albert DM, Pulafito CH (eds) Foundations of ophthalmic pathology. Appleton-Century-Crofts, New York, pp 634-654

4. Wolter JR (1960) The macrophages of the human vitreous body. Am J Ophthalmol 49:99-107

5. Wolter JR (1981) Foveal pathology following anterior segment injury. Ophthalmic Surg 12:661-665

6. Wolter JR (1982) Lens implant cytology. Ophthalmic Surg 13:939-942

7. Wolter JR (1982) Cell life on the surface of lens implants. Graefe's Arch Clin Exp Ophthalmol 218:244-249

8. Wolter JR (1982) Pigment in cellular membranes on intraocular lens implants. Ophthalmic Surg 13:726-732

9. Wolter JR (1982) Leaky cystoid macular edema attracting vitreous macrophages. Ophthalmic Surg 13:568-571

10. Wolter JR (1982) Foveal pathology in ciliary body melanoma. Ophthalmic Surg 13:309-311

11. Wolter JR (1983) Morphology of the capsule-like portion of the reactive membranes on intraocular lens implants. Graefe's Arch Clin Exp Ophthalmol 220:58-65.

12. Wolter JR, Lichter PR (1983) Fibroblast-like cells on intraocular lens implants phagocytizing erythrocytes. $\mathrm{Br} \mathrm{J}$ Ophthalmol (in press)

13. Yanoff M, Fine BS (1982) Ocular pathology, Second Edition. Harper and Row, Philadelphia, pp 590-601

Received April 5, 1983 